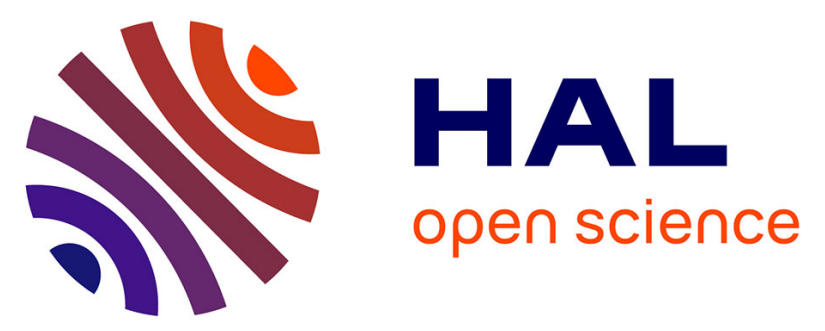

\title{
Adaptive Packet Scheduling for the Support of QoS over DVB-S2 Satellite Systems
}

Elizabeth Rendon-Morales, Jorge Mata-Díaz, Juanjo Alins, José Luis Muñoz, Oscar Esparza

\section{- To cite this version:}

Elizabeth Rendon-Morales, Jorge Mata-Díaz, Juanjo Alins, José Luis Muñoz, Oscar Esparza. Adaptive Packet Scheduling for the Support of QoS over DVB-S2 Satellite Systems. 9th Wired/Wireless Internet Communications (WWIC), Jun 2011, Vilanova i la Geltrú, Spain. pp.15-26, 10.1007/978-3-642-215605_2. hal-01583628

\author{
HAL Id: hal-01583628 \\ https://hal.inria.fr/hal-01583628
}

Submitted on 7 Sep 2017

HAL is a multi-disciplinary open access archive for the deposit and dissemination of scientific research documents, whether they are published or not. The documents may come from teaching and research institutions in France or abroad, or from public or private research centers.
L'archive ouverte pluridisciplinaire HAL, est destinée au dépôt et à la diffusion de documents scientifiques de niveau recherche, publiés ou non, émanant des établissements d'enseignement et de recherche français ou étrangers, des laboratoires publics ou privés. 


\title{
Adaptive packet scheduling for the support of QoS over DVB-S2 satellite systems
}

\author{
Elizabeth Rendon-Morales*, Jorge Mata-Díaz, Juanjo Alins, José Luis Muñoz and \\ Oscar Esparza \\ Departamento de Ingeniería Telemática \\ Universidad Politécnica de Cataluña \\ C. Jordi Girona, 31. 08034 Barcelona. Spain. \\ *elizabeth.rendon@entel.upc.edu
}

\begin{abstract}
This paper presents an adaptive algorithm for managing the weights of a weighted round robin (WRR) scheduler. The weights calculation depends on the capacity variations present in a Digital Video Broadcasting-Second Generation (DVB-S2) satellite link. The algorithm optimizes the bandwidth utilization while satisfying the QoS requirements for different traffic classes. The operation of the proposed algorithm is demonstrated by using the NS-2 simulator environment. The results show that the proposed adaptive WRR algorithm optimizes the bandwidth utilization while enforcing the priority level of each service class even in an extreme reduction of bandwidth caused by rain events.
\end{abstract}

Key words: QoS, DiffServ DVB-S2, BSM and WRR

\section{Introduction}

Nowadays, the support of IP-based applications over Internet has experienced a fast and continuous growth together with the expansion of the World Wide Web. As a consequence, new IP applications such as Voice over IP (VoIP) and multimedia services require considering different levels of individual packet treatment through the network. This differentiation must include not only the Quality of Service (QoS) parameters to specify packet transmission priorities across the network nodes but also, the required amount of bandwidth assignment to guarantee its transport.

To provide this type of IP services, the geostationary (GEO) satellite infrastructure plays a crucial role. Specially, because the satellite systems can provide ubiquity and broadband access, being feasible to reach large and disperse populations around the world.

However, to provide a variety of services over satellite systems with QoS, it is important to consider the intrinsic characteristics present in GEO satellite systems that 
affect the QoS provisioning. Such characteristics are: delay, losses and link bandwidth variations. The presence of these link bandwidth variations need to be seriously considered, as it is necessary to preserve the QoS levels when rain events in the atmosphere are experienced, which can also reduce the available capacity in the Digital Video Broadcasting-Second Generation (DVB-S2) [1] forward channel. To address this, the DVB-S2 specification implements the Adaptive Code and Modulation (ACM) techniques to achieve quasi-error free channel conditions for each individual user, by providing them with the most suitable modulation and code $(\mathrm{Mod} C o d)$ value according to the measured signal-to-noise-plus-interference ratio (SNIR). In addition, the Broadband Satellite Multimedia (BSM) working group has defined a QoS functional architecture (ETSI-BSM-QoS) standardized in [2]. This framework establishes the required QoS mechanisms (supported in the Hub) to define the priorities among users and applications based on the Differentiated Services (DiffServ) architecture [3].

Nevertheless, most of the predefined QoS mechanisms usually have static input parameters resulting in an inefficient resource allocation. Therefore, a solution is to use an adaptive approach that manages the bandwidth distribution (among service classes) considering the fact that the available capacity present in a satellite system is constantly changing. This adaptive approach enables sharing bandwidth resources in a more suitable way, ensuring that the required QoS levels are maintained while the satellite link capacity is optimized.

In this paper, we propose a resource sharing model that manages the QoS requirements and at the same time optimizes the utilization of the DVB-S2 satellite link capacity. The aim of this model is to share the available bandwidth between various service classes having a certain QoS level. The proposed adaptive scheme calculates the optimal weight values considering two factors used to determine the minimum required amount of resources, these are: the QoS requirements of each traffic class and the link bandwidth availability present on the satellite system.

The proposed adaptive IP packet scheduling mechanism is based on the Weighted Round Robin (WRR) scheme which is evaluated using the NS-2 simulation tool. In the simulation environment we consider both the adoption of a DiffServ model to provide QoS guarantees and the most representative characteristics affecting the DVB-S2 forward channel (i.e. delay, losses and mainly the presence of link bandwidth variations). The presented simulation results demonstrate that when our adaptive WRR mechanism is implemented, an enhanced satellite performance is achieved while maintaining the QoS levels even though a severe reduction of bandwidth is experienced.

The presented work is organized in 5 sections: in section 2 a characterization of the bandwidth link variation is presented together with a description of the adaptive Hub and the adaptive WRR algorithm. In section 3, the proposed adaptive model is evaluated using the NS-2 simulation tool and the performance evaluation results are presented. In section 4 the related studies are presented and conclusions are formulated in section 5 . 


\section{Adaptive WRR Model}

In this section we present a characterization of the bandwidth link variations present in GEO satellite systems, a description of the adaptive Hub and the proposed adaptive WRR algorithm.

\subsection{Characterization of the bandwidth link variation}

In practice, transmission rate variations in the satellite link are mainly caused by atmospheric conditions such as rain events. In order to define an adaptive WRR model that considers these link bandwidth variations, we have analyzed a rain event affecting the bandwidth availability in the DVB-S2 forward channel.

Particularly, we have considered the study presented in [4] that provides an estimation of the physical layer performance over the DVB-S2 forward link under a heavy rain event. By considering this approach, the physical layer behavior is adapted taking into account the effects of the satellite delay and the implementation of the ACM techniques. Using such techniques, it is possible to achieve an optimal operation point by selecting the most suitable ModCod depending on the satellite terminal propagation conditions. To simplify and use these results in our proposed adaptive model we approximated the physical layer estimation reported in [4] by a sinusoidal wave (see Fig. 1-dotted line) to represent a rain event in the satellite DVBS2 link.

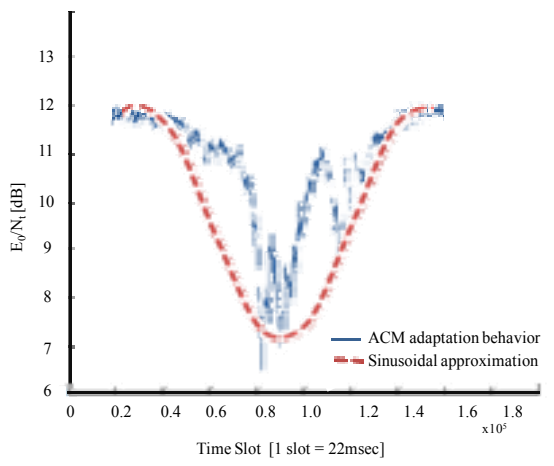

Fig. 1 ACM adaptation behavior during a heavy rain event in the forward link [4]

In this way, satellite terminals allocated under clear sky conditions are characterized for having high link bandwidth availability (see Fig. 1 at the peak of the sinusoidal link wave). Therefore, those terminals will be provided with an efficient ModCod according to the satellite propagation conditions. In contrast, terminals allocated under a heavy rain event are characterized for having reduced link bandwidth availability (see Fig. 1 at the valley of the sinusoidal link wave). Therefore those terminals will be provided with a ModCod including a robust channel protection mechanism and a specific modulation scheme. As a result, the bandwidth capacity will fluctuate between the minimum (valley) and maximum (peak) values, since the ACM 
mechanism will be continuously working to mitigate the atmospheric impairments. This bandwidth characterization will be the input parameter of our proposed IP adaptive scheduler.

\subsection{Architecture of the adaptive Hub}

The proposed DiffServ model implemented over the adaptive DVB-S2 Hub fulfills the ETSI QoS Broadband Satellite Multimedia Services and Architectures standard [2]. The QoS policy implemented in the DiffServ model allows the EF traffic class to have the highest priority, while the $\mathrm{AF}$ traffic class has more priority than the $\mathrm{BE}$ traffic class. As a results, the BE traffic class, which is based on a best effort scheme, uses the remaining link capacity, being able to use the bandwidth that any other class does not use.

The architecture of the adaptive Hub that supports the DiffServ model and exploits our adaptive packet scheduler based on the WRR mechanism is shown in Fig. 2. The DiffServ model includes a traffic classifier that decides if a packet needs to be reassigned with a different QoS level by remarking its DSCP. Notice that the proposed scheme allows multiple flows to be aggregated and treated as a single flow per traffic class. The enqueuing system supported at the Hub has three traffic classes allowing each of them to have its own physical queue and implementing a drop tail mechanism.

Every traffic class implements a token bucket (TB) as a rate limiter to guarantee the transmission rate according to the bandwidth assignment established in the Service Level Agreement (SLA). Each TB limiting rate is set to $\mu_{\mathrm{EF}}, \mu_{\mathrm{AF}}$ and $\mu_{\mathrm{BE}}$ for the EF, $\mathrm{AF}$ and $\mathrm{BE}$ traffic classes respectively. Here, it is worth mentioning that our proposal considers the buffer length of each traffic class as a function of the Bandwidth Delay Product (BDP). This BDP value is set considering the minimum Round Trip Time $\left(\mathrm{RTT}_{\mathrm{MIN}}\right)$ present in the satellite system and the associated TB limiting rate.

All packets coming from each traffic class are sent directly to the IP scheduler. The IP scheduler is based on the Weighted Round Robin (WRR) mechanism [5] that controls the order in which packets are extracted out from its queues. Along with the WRR scheduler, there is a new module which is responsible for calculating the parameters of the WRR scheduler. This component is referred as the Weights calculator that takes into account the adaptive WRR model (further defined in subsection 2.3) to compute the suitable weight values. The Weights calculator's input parameter is the bandwidth availability considering the sinusoidal wave variation defined in subsection 2.1. Such value is sent from the physical layer to the network layer by means of a cross-layer mechanism.

The corresponding weight values $\left(\mathrm{W}_{\mathrm{EF}}, \mathrm{W}_{\mathrm{AF}}\right.$ and $\left.\mathrm{W}_{\mathrm{BE}}\right)$ are set within the IP scheduler to prioritize the resources for each traffic class. As it is observed, in Fig. 2 the Weights calculator module is decoupled from the IP scheduler. Therefore, the scheduler complexity is not increased and both modules can work independently based on their own settings. 


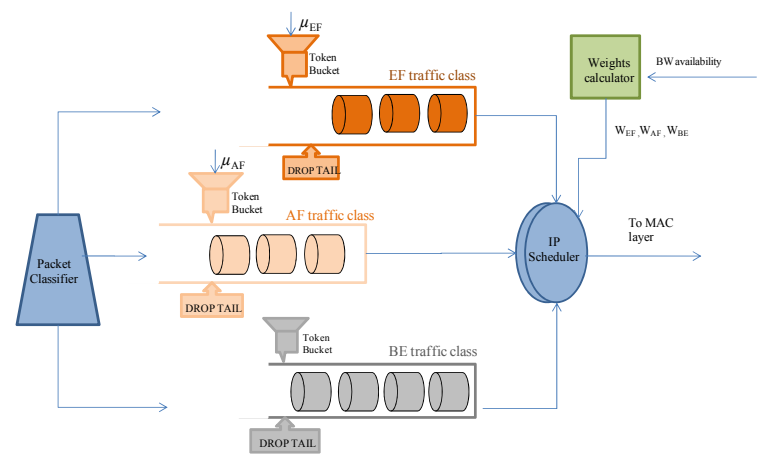

Fig. 2 Structure of the adaptive DVB-S2 Hub

\subsection{The adaptive WRR algorithm}

In order to calculate the weight values, different criteria can be used (e.g. the mean packet size [6], queue sizes [7], revenue-based [8]). In this paper, we propose to use the current capacity of the DVB-S2 satellite link $\left(\mathrm{C}_{\mathrm{OUT}}\right)$ as the main criteria for the allocation of network resources. To the best of our knowledge, this is the first time that such scenario has been analyzed over a DVB-S2 satellite system.

The adaptive IP scheduler design proposed in this paper actively adjusts the weight of each traffic class to enforce its QoS level. This adaptive design is linked to the ACM mechanism specification. In this way, considering the three traffic classes defined in the DiffServ architecture (EF, $\mathrm{AF}$ and $\mathrm{BE})$, the adaptive model will therefore prioritize the $\mathrm{EF}$ traffic class over the $\mathrm{AF}$ and $\mathrm{BE}$ traffic classes when the satellite system experiences a capacity reduction in the presence of a heavy rain event. This prioritization is done by assigning high weight values to the EF traffic class while the AF and BE traffic classes will have the lowest weight values. Similarly, when the intensity of the rain event has diminished and the link capacity increases, the adaptive model will keep the priority levels of the $\mathrm{EF}$ and $\mathrm{AF}$ traffic classes over the $\mathrm{BE}$ traffic by assigning high weight values to the $\mathrm{EF}$ and $\mathrm{AF}$ traffic classes while the $\mathrm{BE}$ traffic class will have the lowest weight value. Finally when the rain event has ended and the bandwidth availability is enough to guarantee the high priority traffic (EF and $\mathrm{AF}$ ), the assigned bandwidth for the BE traffic class will be reestablished and the weight values will be assigned according to the rate of the high priority classes.

To adaptively distribute the available capacity among each traffic class, two thresholds are defined: the LowerCap ${ }_{\mathrm{THR}}$ and the UpperCap ${ }_{\mathrm{THR}}$. The LowerCap ${ }_{\mathrm{THR}}$ is set to the EF limiting rate $\left(\mu_{\mathrm{EF}}\right)$ specifying when the system capacity $\left(\mathrm{C}_{\mathrm{OUT}}\right)$ is scarce but is still enough to guarantee the EF traffic class. The upper threshold UpperCap ${ }_{\text {THR }}$ is set to the sum of the $\mathrm{EF}$ and $\mathrm{AF}$ limiting rates $\left(\mu_{\mathrm{EF}}+\mu_{\mathrm{AF}}\right)$ specifying when the system capacity is enough to guarantee the rate of both priority classes. If the bandwidth capacity is kept beyond the UpperCap ${ }_{T H R}$, it is considered that the available bandwidth in the system is broad enough to transport all the traffic classes. 
In order to specify such functionality on an adaptive WRR scheduler, Fig. 3 shows the weight values at each interval as a function of the DVB-S2 system capacity $\left(\mathrm{C}_{\text {OUT }}\right)$.

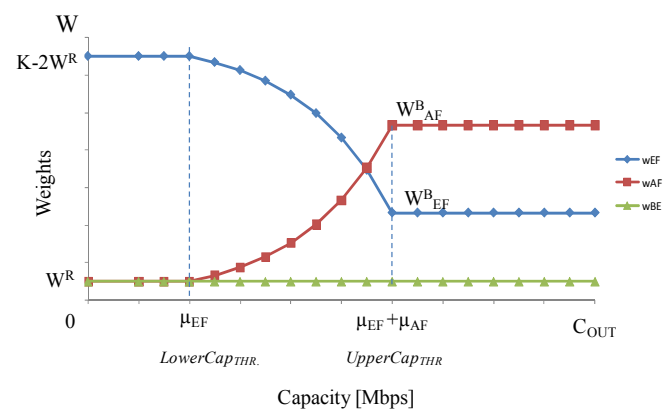

Fig. 3 The weight values as a function of the DVB-S2 system capacity ( $\left.\mathrm{C}_{\text {OUT }}\right)$.

Here, the sum of the weights is set to $\mathrm{K}$, where $\mathrm{K}$ is considered as a constant value that must be significantly high to avoid weight rounding errors:

$$
\mathrm{W}_{\mathrm{EF}}+\mathrm{W}_{\mathrm{AF}}+\mathrm{W}_{\mathrm{BE}}=\mathrm{K}
$$

Given that the BE traffic class has the lowest priority level, our adaptive model allocates the excess of resources in this class. Therefore, in all cases $W_{B E}$ is set to $\mathrm{W}^{\mathrm{R}}$ representing a residual and constant weight value.

$$
\mathrm{W}_{\mathrm{BE}}=\mathrm{W}^{\mathrm{R}}
$$

As it is shown in Fig. 3 when the system capacity is broad enough, $\mathrm{C}_{\text {OUT }}>$ UpperCap $_{\text {THR }}$, our adaptive model assigns constant weight values to guarantee high priority classes (EF and $\mathrm{AF}$ ). We assume that the weight values of each traffic class are set to $\mathrm{W}_{E F}^{\mathrm{B}}$ and $\mathrm{W}^{\mathrm{B}}$ for the high priority classes and $\mathrm{W}^{\mathrm{R}}$ for the $\mathrm{BE}$ class. Following this assumption the next relationship is obtained:

$$
\mathrm{W}_{\mathrm{AF}}^{\mathrm{B}}+\mathrm{W}_{\mathrm{EF}}^{\mathrm{B}}=\mathrm{K}-\mathrm{W}^{\mathrm{R}}
$$

To proportionally distribute the system resources [9], the ratio of $\mathrm{W}^{\mathrm{B}}{ }_{\mathrm{EF}}$ over $\mathrm{W}^{\mathrm{B}}{ }_{\mathrm{AF}}$ is set equal to the ratio of the associated TB limiting rates, thus:

$$
\frac{\mathrm{W}_{\mathrm{EF}}}{\mathrm{W}_{\mathrm{AF}}^{\mathrm{B}}}=\frac{\mu_{\mathrm{EF}}}{\mu_{\mathrm{AF}}}
$$

As a result, the $\mathrm{W}_{\mathrm{EF}}^{\mathrm{B}}$ and $\mathrm{W}^{\mathrm{B}}{ }_{\mathrm{AF}}$ values can be expressed as a function of the $\mathrm{TB}$ limiting rates $\left(\mu_{\mathrm{EF}}\right.$ and $\left.\mu_{\mathrm{AF}}\right)$ as:

$$
\begin{aligned}
& \mathrm{W}_{\mathrm{AF}}^{\mathrm{B}}=\frac{\mathrm{K}-\mathrm{W}^{\mathrm{R}}}{\left(\mu_{\mathrm{EF}+} \mu_{\mathrm{AF}}\right)} \mu_{\mathrm{AF}} \\
& \mathrm{W}_{\mathrm{EF}}=\frac{\mathrm{K}-\mathrm{W}^{\mathrm{R}}}{\left(\mu_{\mathrm{EF}+} \mu_{\mathrm{AF}}\right)} \mu_{\mathrm{EF}}
\end{aligned}
$$

In a similar way, when the system capacity is scarce, $\mathrm{C}_{\mathrm{OUT}}<$ LowerCap $_{\mathrm{THR}}$, our adaptive model assigns constant weight values to guarantee the EF priority class over 
the $\mathrm{AF}$ and $\mathrm{BE}$ traffic classes. Therefore, $\mathrm{W}_{\mathrm{EF}}, \mathrm{W}_{\mathrm{AF}}$ and $\mathrm{W}_{\mathrm{BE}}$ are set as:

$$
\begin{gathered}
\mathrm{W}_{\mathrm{AF}}=\mathrm{W}_{\mathrm{BE}}=\mathrm{W}^{\mathrm{R}} \\
\mathrm{W}_{\mathrm{EF}}=\mathrm{K}-2 \mathrm{~W}^{\mathrm{R}}
\end{gathered}
$$

Finally, to guarantee the EF traffic class over the AF traffic class, when the system capacity is constrained, LowerCap $\mathrm{p}_{\mathrm{THR}}<\mathrm{C}_{\mathrm{OUT}}<\mathrm{UpperCap}_{\mathrm{THR}}$, our adaptive model allocates most of the resources to the EF traffic class. At this interval, we can apply either a linear or an exponential relationship between the weights of the higher priority classes $\left(\mathrm{W}_{\mathrm{EF}}\right.$ and $\left.\mathrm{W}_{\mathrm{AF}}\right)$ and the satellite link capacity $\left(\mathrm{C}_{\mathrm{OUT}}\right)$.

We have performed a practical analysis between the high priority weights and the available bandwidth at the constrained interval. Such analysis has enabled us to conclude that the weight variations must change in a progressive way following an exponential function. However, we have also analyzed the case when a linear weight function is used, resulting in a faster variation where it is not possible to guarantee the EF priority traffic class when the available capacity is close to the UpperCap ${ }_{\text {THR }}$. Therefore, in order to build a continuous weight function for each traffic class, we define $\mathrm{W}_{\mathrm{AF}}$ as follows:

$$
\mathrm{W}_{\mathrm{AF}}=\mathrm{W}^{\mathrm{R}}\left[\frac{\mathrm{W}_{\mathrm{AF}}^{\mathrm{B}}}{\mathrm{W}^{\mathrm{R}}}\right]^{\frac{\mathrm{C}_{\text {out }}-\mu_{\mathrm{FF}}}{\mu_{\mathrm{AF}}}}
$$

Similarly, the $\mathrm{W}_{\mathrm{EF}}$ is obtained as follows:

$$
\mathrm{W}_{\mathrm{EF}}=\mathrm{K}-\mathrm{W}^{\mathrm{R}}-\mathrm{W}_{\mathrm{AF}}
$$

\section{Performance Evaluation}

In this section the satellite network settings and performance metrics are presented. Then, the proposed adaptive WRR model is simulated over a DVB-S2 satellite network and compared with the Round Robin (RR) and the ordinary WRR scheduler.

\subsection{Satellite network settings}

The simulation is carried out employing the NS-2 simulator tool version 2.29. For QoS purposes the DiffServ module was developed using the library described in [10] where our adaptive WRR model has been added. The network topology used for the simulation is shown in Fig. 4. Here, three sources send data to a remote destination. Each source node supports different services, having a predefined PHB. The EF class is represented by a real-time Voice over IP (VoIP) application employing the User Data Protocol (UDP). The AF class represents Hypertext Transfer Protocol (HTTP) 
traffic. The BE class represents bulk data transfer generated by a persistent File Transfer Protocol (FTP) transaction server. The transport protocol used to transport the AF and BE traffic classes is the Sack [11] variant of the Transmission Control Protocol (TCP). We used the TCP Linux version [12], which includes this TCP variant. The details of each service class are presented on Table I.

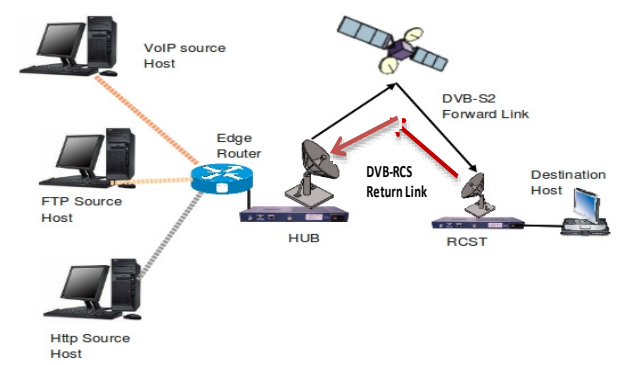

Fig. 4 DiffServ-satellite network topology

All the traffic entering the edge router is directly sent to the adaptive Hub that supports the DiffServ architecture to guarantee the predefined QoS level for each traffic type. The Hub, which is the main element in the satellite system, is responsible for applying the adaptive WRR model and sending the data to the destination host via the DVB-S2 forward channel. The return channel is used to transport signaling data, based on the DVB-RCS specification [13]. The DVB-S2 satellite network settings used throughout the simulation are configured as follows:

The satellite channel capacity considered as the bottleneck link is set considering the terminals in clear sky conditions will have $3.4 \mathrm{Mbps}$ of bandwidth while terminals under a rain event will have a reduced capacity set to $0.4 \mathrm{Mbps}$. Therefore, the sinusoidal link variation will fluctuate between 0.4 to $3.4 \mathrm{Mbps}$. The minimum Round Trip Time $\left(\mathrm{RTT}_{\mathrm{MIN}}\right)$ experienced by the satellite system is set to $560 \mathrm{msec}$. The buffer length of each traffic class is set to the BDP value. Particularly, the buffer length of the BE traffic class is set to 90 packets. The Packet Error Rate (PER) is set to 1x10-7.

The transfer speed for each traffic class considered for the simulation is: $400 \mathrm{Kbps}$ for the EF traffic class and $800 \mathrm{Kbps}$ for the AF traffic class, while the BE traffic class will use the remaining bandwidth. The LowerCap ${ }_{\mathrm{THR}}$ and the UpperCap $\mathrm{T}_{\mathrm{THR}}$ values are set to $0.4 \mathrm{Mbps}$ and $1.2 \mathrm{Mbps}$ respectively.

Table 1. Parameters of each service class

\begin{tabular}{ccccc}
\hline Traffic Class & Max Flows & Rate per-class [Kbps] & Packet Size [bytes] & C OUT $_{\text {OMbps] }}$ \\
\hline EF & 10 & 400 & 1500 & $0.4-3.4$ \\
AF & 10 & 800 & 1500 & $0.4-3.4$ \\
BE & 10 & 3400 & 1500 & $0.4-3.4$ \\
\hline
\end{tabular}




\subsection{Performance metrics}

The proposed analysis is carried out at the bottleneck-satellite link, therefore, the relevant TCP information such as: the transmitted/acknowledged packets, the congestion window and the RTT are collected at the sender's side. Additionally, in order to asses the system performance with the proposed adaptive WRR scheduler, two metrics are considered: the goodput and the buffer occupancy.

The goodput is defined as the average amount of correctly received data (excluding retransmissions) measured over a certain period of time. The goodput per-class is calculated by dividing the amount of transmitted data by the number of active data flows (10 flows) within a service class in a given interval.

The buffer occupancy represents the fullness of the DiffServ queue during the simulation time. In our case, the simulation results consider the buffer occupancy for the IP queues allocated at the Hub.

\subsection{Simulation results}

In order to evaluate the performance of the proposed adaptive scheduler, three simulation tests are considered: the first test is performed to assess the DVB-S2 system response when the Round Robin (RR) mechanism is employed. The second test considers the Weighted Round Robin mechanism with static weight values set to 6,8 and $1(\mathrm{~K}=15)$ for $\mathrm{W}_{\mathrm{EF}}, \mathrm{W}_{\mathrm{AF}}$ and $\mathrm{W}_{\mathrm{BE}}$ respectively. These values are set considering the proportional distribution of resources, where the EF traffic class has higher weight value than its corresponding proportional value. Finally, a third simulation test is performed considering our proposed adaptive WRR scheduler to adjust the weight values taking into account the capacity variations. Here, we have calculated the weight values at the constrained interval considering both functions: the linear weight function and the exponential weight function. The results of plotting the dynamics of weights as a function of the sinusoidal link variation are shown in Fig. 5.

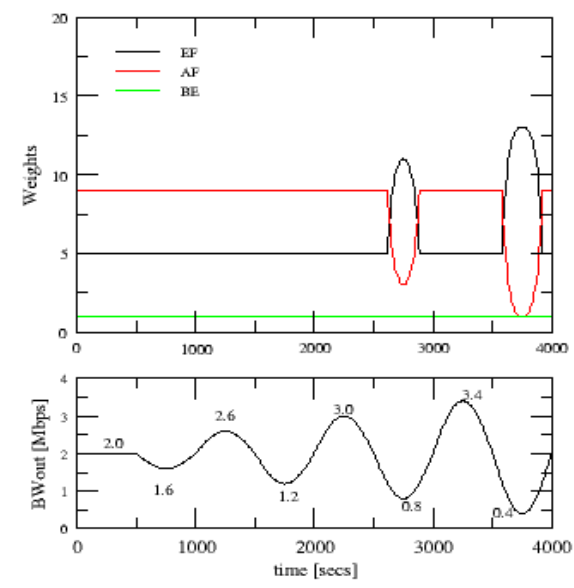

Fig. 5. Dynamics of Weights 
In this case, the link bandwidth variation $\left(\mathrm{C}_{\text {OUT }}\right)$ oscillates between 0.4 and 3.4 Mbps. These results consider the exponential adaptive WRR algorithm (using the analysis presented in section 2.3) and the bandwidth rate previously set for each traffic class. The $\mathrm{K}$ value is set to 15 and $\mathrm{W}^{\mathrm{R}}$ is set to 1 .

Fig. 6a illustrates the simulation results of goodput and queue occupancy considering the RR algorithm. Using this algorithm it is observable that the EF and AF traffic classes are not guaranteed when a reduction of bandwidth (due to a rain event) is experienced. Moreover, the queue occupancy is overloaded in most of the cases leading to an increase of the system latency.

In a similar way, by using the WRR mechanism (see Fig. 6b) with static weight values, the EF traffic class is able to reach its $400 \mathrm{Kbps}$. However, its goodput is affected in specific time points when the capacity is reduced (see the valleys of the sine wave).
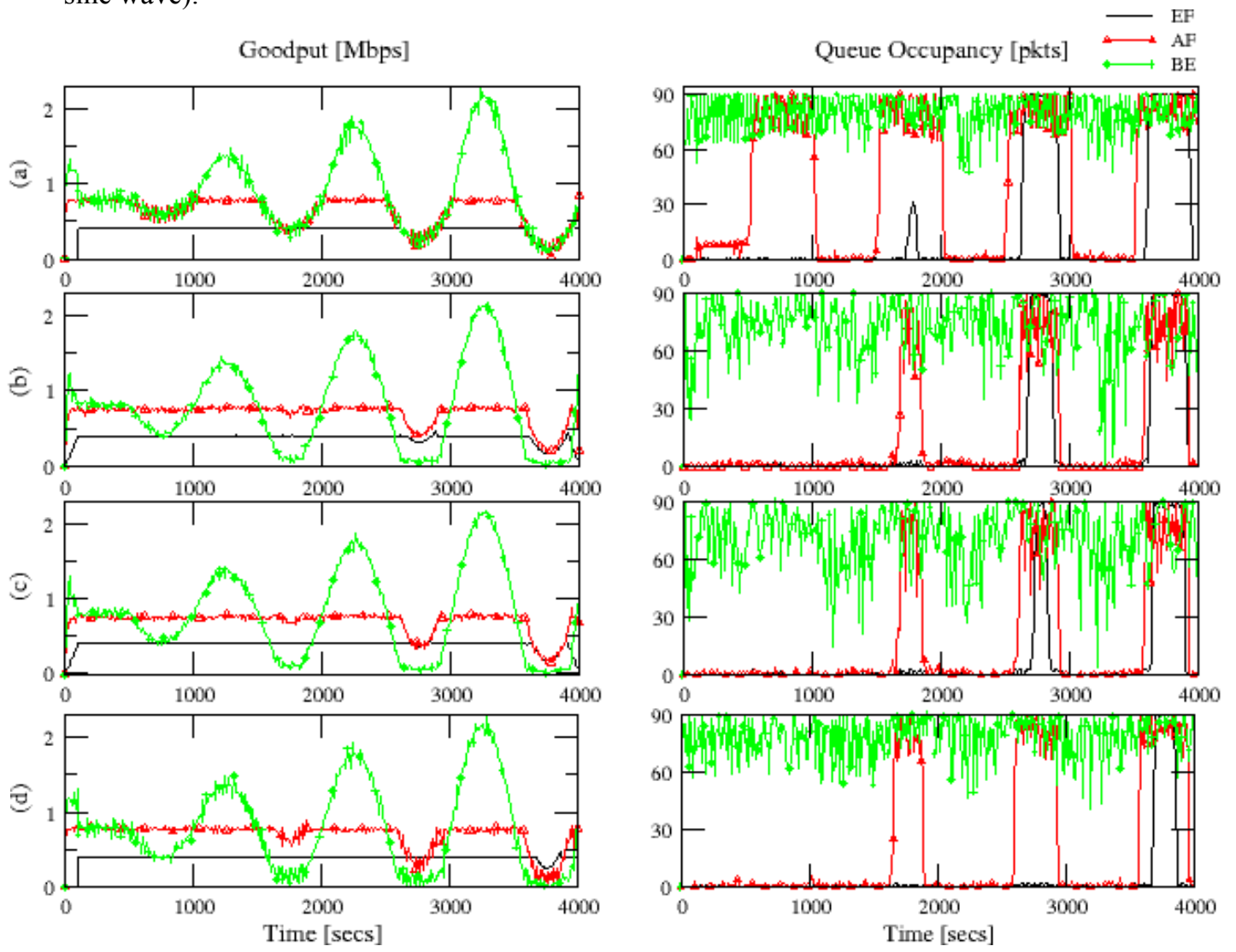

Fig. 6. Goodput performance results and queue occupancy with different scheduler types: (a) Round Robin mechanism, (b) Weighted Round Robin, (c) Linear Adaptive WRR and (d) Exponential Adaptive WRR 
Likewise, the AF traffic class tries to keep its $800 \mathrm{Kbps}$ of goodput, while the $\mathrm{BE}$ traffic class uses the remaining bandwidth. The buffer occupancy (considering the three traffic classes) is overloaded, allowing an increase of the system latency which is reduced compared to the latency experienced in the RR scenario. It is also observable that when an extreme reduction of bandwidth is experienced (see the interval between 3500-4000 sec) the priority levels of each traffic class are lost, being unable to guarantee the $\mathrm{EF}$ traffic class over the $\mathrm{AF}$ and $\mathrm{BE}$ traffic classes.

Similarly, by using the linear adaptive WRR mechanism (Fig. 6c), it is observable that the EF buffer occupancy is reduced compared to the previous case. However, given that a linear function generates a faster weight variation, it is not possible to guarantee the EF priority traffic class when the available capacity is reduced (see the interval between 3500-4000 sec).

Finally, considering our proposed exponential adaptive WRR mechanism (Fig. 6d) it is observable that the buffer occupancy for the EF traffic class is kept at lower levels and that the rate for the EF traffic class is totally guaranteed (400 Kbps) even though an extreme reduction of bandwidth is experienced. In addition, the transmission rate for the $\mathrm{AF}$ traffic class reaches its $800 \mathrm{Kbps}$ of goodput and the $\mathrm{BE}$ traffic class uses the remaining bandwidth, being able to follow the sine wave when an increase of bandwidth is experienced by the system. It is also observable that when an extreme reduction of bandwidth is experienced (see the interval between 3500$4000 \mathrm{sec}$ ) the priority levels of each traffic class are kept, guaranteeing the EF traffic class over the AF and BE traffic classes.

\section{Related works}

In order to calculate the optimal weight values based on the QoS requirements, different criteria have been researched in the literature e.g. the mean packet size, queue sizes, revenue-based etc.

In [6], a Variable WRR (VWRR) scheduler is proposed. This model uses the average packet length to adapt its weights. A weight value is calculated considering two parameters: the bandwidth requirements and the average length of packets. The problem of adjusting weights for the WRR scheduler guaranteeing the premium service has been analyzed in [7]. This work proposes a resource allocation model considering the average queue size, which is calculated using a low-pass filter. In [8], an adaptive scheduling scheme using the revenue-based WRR is presented. This proposal has been considered for guaranteeing the maximum service provider revenue. The proposed scheme, adjusts its weights using the revenue criteria to control the resource allocation. In [14], a modified Fair WRR (FWRR) scheduler has been proposed to protect the best effort traffic from the assured forwarding out-ofprofile packets in the core routers based on the DiffServ architecture. This policy dynamically adjusts the weights and the buffer allocation using congestion hints to avoid unfair bandwidth sharing. 


\section{Conclusions}

We have presented an adaptive resource management algorithm based on a WRR scheduler. This proposed algorithm uses the link bandwidth variations present in the DVB-S2 forward link to determine the minimum amount of resources for each traffic class considering the DiffServ architecture. To calculate the optimal weight values, we have applied two functions to associate the weights of the higher priority classes and the satellite link capacity: a linear and an exponential weight function. By employing the NS-2 simulator tool we have demonstrated that using our adaptive WRR algorithm it is possible to ensure the QoS requirements for each traffic class and to optimize the bandwidth utilization when bandwidth fluctuations caused by rain events are faced. The simulation results have shown that our exponential adaptive WRR model enforces the priority levels and guarantees the QoS parameters established in the SLA, while maintaining the queue occupancy at lower levels (compared to the RR and WRR mechanism), leading to a reduction of the system latency.

\section{References}

1. ETSI ES 302 307, ETSI Standard for Digital Video Broadcasting (DVB); Second generation framing structure, channel coding and modulation systems for Broadcasting, Interactive Services, News Gathering and other broadband satellite applications (DVB-S2). 2004.

2. ETSI TS 102 462, ETSI Standard for Satellite Earth Stations and Systems (SES); Broadband Satellite Multimedia (BSM); QoS Functional Architecture. 2006.

3. IETF RFC 2475, Standard for an Architecture for Differentiated Services, December 1998.

4. S. Cioni, R.Gaudency, and R.Rinaldo."Channel estimation a physical layer adaptation techniques for satellite networks exploiting adaptive coding and modulation". International Journal of Satellite communication and networking, vol. 26 no. 2, 2008, pp. 157-188.

5. H. Shimonishi and H. Suzuki, "Performance Analysis of Weighted Round Robin Cell Scheduling and Its Improvement in Atm Networks," IEICE Transactions on Communications. vol. E81B, no. 5, 1998, pp. 910-918.

6. Y. Ito, S. Tasaka and Y. Ishibashi, "Variably weighted round robin for core IP networks" Performance, computing and communication conference, 2002, pp. 159-166.

7. H. Wang, C. Shen and S.K.G. "Adaptive-weighted packet scheduling for premium service". IEEE International Conference on Communications, vol. 6, 2001, pp. 1846-1850

8. A. Sayenko, T. Hamalainen and P. Raatikainen "Adaptive scheduling using the revenuebased weighted round robin". IEEE International Conference on Networks, 2004, pp.743-49.

9. C. Dovrolis and P. Ramanathan, "A case for relative Differentiated Services and Proportional Differentiation Model”; IEEE Network Magazine, 1999, pp. 26-34.

10. P. Pieda, J. Ethridge, M. Bainess and F. Shallwani "A Network Simulator Differentiated Services Implementation”. Open IP Nortel Networks, 2000.

11. IETF RFC 3517. IETF Standard for a Conservative Selective Acknowledgment (SACK)based. Loss Recovery Algorithm for TCP, 2003

12. D.Wei and P. Cao, "A Linux TCP implementation for NS2", available at the Internet, 2006.

13. ETSI EN 301 790, ETSI Standard for Digital Video Broadcasting (DVB); Interaction Channel for Satellite Distribution Systems; (DVB-RCS), 2005.

14. S. Yi, X. Deng, G. Kesidis and C. R. Das "Providing fairness in DiffServ architecture"; IEEE Global Telecommunications Conference, vol. 2, 2002, pp. 1435-1439. 